\title{
INFLUÊNCIA DAS VARIÁVEIS OPERACIONAIS NA REMOÇÃO DE ETANOL DE VINHO DELEVEDURADO POR $\mathrm{CO}_{2}$
}

\author{
C. R. SILVA ${ }^{1}$, M. N. ESPERANÇA ${ }^{1}$, A. J. G. CRUZ ${ }^{1}$ e A. C. BADINO ${ }^{1}$ \\ ${ }^{1}$ Universidade Federal de São Carlos, Departamento de Engenharia Química \\ E-mail para contato: camilaramos@gvo.com.br
}

\begin{abstract}
RESUMO - Na fermentação alcoólica, o fenômeno da inibição pelo produto limita a concentração de etanol no vinho ao redor de $10^{\circ} \mathrm{GL}$, o que resulta em grande volume de vinhaça (ca. $12 \mathrm{~L}_{\text {vinhaça }} / \mathrm{L}_{\text {etanol }}$ ) e alto consumo de vapor na etapa de destilação (ca. $2,5 \mathrm{~kg}_{\text {vapor }} / \mathrm{L}_{\text {etanol }}$ ). Tecnologias para remover etanol do vinho vêm sendo estudadas. Exemplo é a operação de arraste de etanol por um gás, que além da remoção de etanol, provoca também a redução da temperatura do meio reacional devido à sua vaporização. Este fato é vantajoso, pois o controle da temperatura nas dornas industriais é um fator de difícil solução por envolver grandes volumes. Neste contexto, o arraste de etanol por gás pode ser utilizado tanto no controle da concentração de etanol e na redução da temperatura do meio fermentativo, como pode ser utilizado após a fermentação para a extração de etanol do vinho, sendo uma alternativa à destilação, reduzindo o consumo de vapor da planta. Neste trabalho, avaliou-se o arraste de etanol do vinho delevedurado por $\mathrm{CO}_{2}$ (gerado no próprio processo industrial) em dorna de $95 \mathrm{~L}$. Um planejamento fatorial foi utilizado para avaliar a influência das variáveis, temperatura inicial do vinho $\left(\mathrm{T}_{\mathrm{o}}\right)$ e vazão específica de $\mathrm{CO}_{2}$ $\left(\Phi_{\mathrm{CO} 2}\right)$ nos parâmetros de desempenho: Fator de Arraste $\left(\mathrm{F}_{\mathrm{A}}\right)$, Fator de Concentração $\left(\mathrm{F}_{\mathrm{C}}\right)$ e Fator de Redução de Temperatura $\left(\mathrm{F}_{\mathrm{T}}\right)$. Observou-se que o $\mathrm{F}_{\mathrm{A}}$ e o $\mathrm{F}_{\mathrm{T}}$ foram influenciados positivamente pelas variáveis $\mathrm{T}_{\mathrm{o}}$ e $\Phi_{\mathrm{CO} 2}$ enquanto o $\mathrm{F}_{\mathrm{C}}$ foi influenciado negativamente, apenas pela variável $\mathrm{T}_{\mathrm{o}}$.
\end{abstract}

\section{INTRODUÇÃO}

No Brasil, a produção de etanol gera ganhos ambientais, sociais e promove o crescimento de forma significativa em mais de mil municípios brasileiros. Além de emitir $90 \%$ menos gases que contribuem para as mudanças climáticas do que a gasolina, a produção do etanol (combustível renovável) a partir da cana-de-açúcar gera oito vezes mais empregos que a indústria do petróleo (UNICA), e também ajuda a reduzir a dependência do petróleo importado. Nas destilarias brasileiras são gerados em torno de $12 \mathrm{~L}_{\text {vinhaça }} / \mathrm{L}_{\text {etanol, }}$, pois o etanol contido no vinho ao final da fermentação atinge valores entre 8 e $9{ }^{\circ} \mathrm{GL}$. Essa concentração de etanol limita a redução dos volumes de vinhaça. Trabalhar com concentrações de etanol no vinho acima de $10^{\circ} \mathrm{GL}$ reduz a viabilidade das leveduras (na temperatura de operação industrial), o que inviabiliza a reciclagem de células. Como as leveduras são reutilizadas várias vezes durante toda a safra (processo MélleBoinot), torna-se importante a manutenção das células (Amorim et al., 2011). Além disso, para ser utilizado como combustível, a concentração de etanol deve ser de pelo menos $94,5^{\circ} \mathrm{GL}$ (ANP). Portanto, o consumo de energia para concentrar o produto obtido na fermentação é significativo 
(Morandim et al., 2011). Na tentativa de superar esta limitação do processo fermentativo, tecnologias alternativas designadas a remover o etanol continuamente da fermentação alcoólica vêm sendo estudadas. Exemplos são os processos de fermentação e extração do etanol simultaneamente que foram estudados: extração a vácuo, que proporciona a remoção de etanol de um processo fermentativo acoplado a um tanque flash (Rivera et al., 2010); extração por solvente, que consiste na remoção contínua do etanol do caldo em fermentação utilizando um solvente adequado (Schugerl, 2000) e operação de stripping, que é um processo físico no qual é possível controlar a concentração de etanol no caldo por meio de sua retirada através do borbulhamento de um gás de arraste (Taylor et al., 1997; Esperança et al., 2012). Taylor et al. (1997) estudaram o processo de fermentação e extração de etanol, utilizando o stripping, sendo o etanol arrastado pelo próprio $\mathrm{CO}_{2}$ produzido durante a fermentação. Os autores encontraram valores de produtividades elevados (14-17 $\left.\mathrm{g}_{\text {etanol }} \cdot \mathrm{L}^{-1} \cdot \mathrm{h}^{-1}\right)$. Esperança et al. (2012) estudaram a retirada de etanol de uma solução hidroalcoólica, utilizando o stripping, empregando-se $\mathrm{CO}_{2}$ como gás de arraste. Os autores concluíram que é possível remover etanol empregando-se a operação de stripping e também, reduzir a temperatura do meio reacional. Este último efeito seria de grande vantagem para as usinas, pois o controle da temperatura das dornas é fator de grande importância, porém bastante complicado, principalmente em dias com temperatura ambiente elevada. Neste contexto, a operação de stripping surge como uma alternativa promissora para superação da limitação da fermentação devido a toxicidade do etanol para as leveduras e para o controle da temperatura do meio durante o processo fermentativo. Além disso, o stripping poderia ser utilizado no vinho delevedurado para extrair o etanol, sendo uma alternativa à destilação, podendo reduzir o consumo de vapor da planta. Os objetivos deste trabalho foram:

(1) Avaliar a remoção de etanol de um vinho delevedurado, através da operação de stripping utilizando dióxido de carbono como gás de arraste, sendo este gerado na própria fermentação alcoólica na proporção de $432 \mathrm{~L}_{\mathrm{CO} 2} / \mathrm{L}_{\text {etanol }}\left(33^{\circ} \mathrm{C}\right.$ e 1 atm);

(2) Verificar a influência das variáveis independentes, temperatura inicial do vinho delevedurado $\left(\mathrm{T}_{\mathrm{o}}\right)$ e vazão específica de $\mathrm{CO}_{2}\left(\Phi_{\mathrm{CO} 2}\right)$, em parâmetros de desempenho como o fator de arraste $\left(\mathrm{F}_{\mathrm{A}}\right)$, fator de redução de temperatura $\left(\mathrm{F}_{\mathrm{T}}\right)$ e fator de concentração $\left(\mathrm{F}_{\mathrm{C}}\right)$.

\section{MATERIAIS E MÉTODOS}

\subsection{Equipamentos e Procedimento Experimental}

Os experimentos foram realizados em uma planta piloto instalada na empresa Virgolino de Oliveira S/A (Unidade Catanduva, SP). Nos ensaios realizados, a temperatura do vinho (T) variou livremente durante o processo de stripping, enquanto que a vazão específica de dióxido de carbono $\left(\Phi_{\mathrm{CO} 2}\right)$ permaneceu constante. Na Figura 1 é apresentado um esquema representativo da planta piloto.

$\mathrm{O}$ gás de arraste (dióxido de carbono) alimentado pela corrente $\mathrm{F}_{1}$ foi capturado no topo da torre de lavagem de gases da unidade industrial, com o auxílio de duas bombas de vácuo ou dois compressores ligados em paralelo (equipamentos $n^{0} 4$ e $n^{0} 5$ ). Inicialmente, os ensaios foram realizados utilizando-se as bombas de vácuo e, posteriormente, com o intuito de aumentar a $\Phi_{\mathrm{CO} 2}$, as bombas foram substituídas pelos compressores. No entanto, como a corrente $F_{1}$ apresentava alta umidade, o que poderia danificar os equipamentos, esta corrente passou, primeiramente, por um 
trocador de calor casco e tubo (equipamento $\mathrm{n}^{\mathrm{o}} 1$ ), onde trocou calor com a água proveniente das torres de resfriamento da unidade industrial (correntes $\mathrm{F}_{2 \text {,entrada }}$ e $\mathrm{F}_{2 \text {,saída }}$ ) e então o $\mathrm{CO}_{2}$ foi enviado para um tanque de recheio (equipamento $\mathrm{n}^{\circ} 2$ ) que também auxiliou na condensação da água existente no gás. A água condensada foi armazenada em um reservatório (equipamento $\mathrm{n}^{\mathrm{o}} 3$ ) e descartada de hora em hora (corrente $\mathrm{F}_{1}$ ”) através de uma válvula. A seguir, o $\mathrm{CO}_{2}$ (corrente $\mathrm{F}_{1}$ ) passou pelas duas bombas de vácuo ou dois compressores (equipamentos $n^{0} 4$ e $n^{0} 5$ ) e, posteriormente, por uma placa de orifício (equipamento $\mathrm{n}^{\mathrm{o}}$ 6), onde a queda de pressão foi determinada por um medidor de pressão eletrônico (equipamento $\mathrm{n}^{0} 7$ ). Dessa forma, tendo-se a queda de pressão e com o auxílio de uma equação de conversão, foi possível determinar a vazão de $\mathrm{CO}_{2}$ enviada para o tanque de vinho (equipamento $\mathrm{n}^{\circ} 8$ ). Nos experimentos foi utilizado um volume inicial de vinho delevedurado de $95 \mathrm{~L}$ e, como gás de arraste, utilizou-se o dióxido de carbono, que foi borbulhado uniformemente no tanque de vinho com ajuda de um distribuidor de gás (equipamento $\mathrm{n}^{\mathrm{o}}$ 9) composto por 6 aletas com 10 furos cada. Além do distribuidor de gás, o tanque de vinho possui um medidor de nível acoplado (equipamento $n^{\circ} 10$ ), serpentina de cobre no interior (equipamento $n^{0} 11$ ), termômetro na entrada da serpentina (equipamento $n^{0} 12$ ) para controlar a temperatura de entrada do líquido de aquecimento composto por flegmaça e água (correntes $\mathrm{F}_{4 \text {,entrada }}$ e $\mathrm{F}_{4 \text {,saída }}$ ), um termômetro digital para acompanhar a temperatura do vinho e a temperatura ambiente (equipamento $n^{\circ} 13$ ) e uma válvula para amostragem (equipamento $n^{0} 14$ ). Cada ensaio teve duração de 8 horas e, durante este período foram monitoradas as seguintes variáveis: concentração de etanol no tanque de vinho, temperatura do vinho, temperatura ambiente, temperatura do dióxido de carbono, volume da solução remanescente no tanque e vazão específica de $\mathrm{CO}_{2}$.

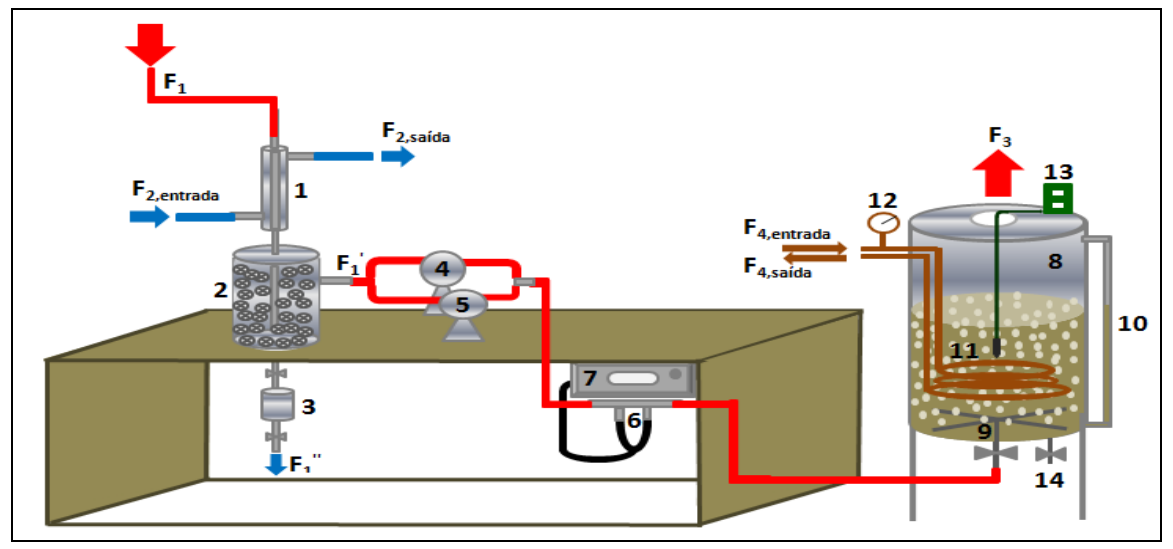

Figura 1 - Esquema representativo da unidade piloto montada na Usina Catanduva.

\subsection{Planejamento Experimental Estatístico e Tratamento dos Dados}

Com o intuito de se identificar as variáveis operacionais mais importantes no processo de stripping, utilizou-se um planejamento fatorial para duas variáveis independentes conforme descrito por Rodrigues e Iemma (2009). Na Tabela 1 estão apresentadas as variáveis independentes, temperatura inicial do vinho $\left(\mathrm{T}_{\mathrm{o}}\right)$ e vazão específica do $\mathrm{CO}_{2}\left(\Phi_{\mathrm{CO} 2}\right)$, e os seus respectivos níveis. Na Tabela 2 estão as matrizes do planejamento fatorial. 
Tabela 1 - Variáveis independentes e seus respectivos níveis.

\begin{tabular}{ccccc}
\hline Variáveis & Código & \multicolumn{3}{c}{ Níveis } \\
& & $\mathbf{- 1}$ & $\mathbf{0}$ & $\mathbf{+ 1}$ \\
\hline $\mathrm{T}_{\mathrm{o}}\left({ }^{\mathrm{o}} \mathrm{C}\right)$ & $\mathrm{X}_{1}$ & 33 & 49 & 65 \\
$\Phi_{\mathrm{CO} 2}(\mathrm{vvm})$ & $\mathrm{X}_{2}$ & 0,10 & 0,20 & 0,30 \\
$\Phi_{\mathrm{CO} 2}(\mathrm{vvm})$ & $\mathrm{X}_{3}$ & 0,50 & 0,75 & 1,00 \\
\hline
\end{tabular}

Tabela 2 - Matrizes do planejamento fatorial.

\begin{tabular}{ccc|ccc}
\hline Ensaio & $\mathbf{X}_{\mathbf{1}}$ & $\mathbf{X}_{\mathbf{2}}$ & Ensaio & $\mathbf{X}_{\mathbf{1}}$ & $\mathbf{X}_{\mathbf{3}}$ \\
\hline $\mathbf{1}$ & -1 & -1 & $\mathbf{8}$ & -1 & -1 \\
$\mathbf{2}$ & -1 & +1 & $\mathbf{9}$ & -1 & +1 \\
$\mathbf{3}$ & +1 & -1 & $\mathbf{1 0}$ & +1 & -1 \\
$\mathbf{4}$ & +1 & +1 & $\mathbf{1 1}$ & +1 & +1 \\
$\mathbf{5}$ & 0 & 0 & $\mathbf{1 2}$ & 0 & 0 \\
$\mathbf{6}$ & 0 & 0 & $\mathbf{1 3}$ & 0 & 0 \\
$\mathbf{7}$ & 0 & 0 & $\mathbf{1 4}$ & 0 & 0 \\
\hline
\end{tabular}

Como variáveis resposta foram calculados os valores do Fator de Arraste $\left(\mathrm{F}_{\mathrm{A}}\right)$, Fator de Redução de Temperatura $\left(\mathrm{F}_{\mathrm{T}}\right)$ e Fator de Concentração $\left(\mathrm{F}_{\mathrm{C}}\right)$, definidos conforme as Equações 1, 2 e 3 .

$$
\begin{aligned}
& F_{A}=\frac{m_{E T_{0}}-m_{E T_{i}}}{m_{E T_{0}}} \times 100=\left(\frac{C_{E T_{0}} \times V_{0}-C_{E T_{i}} \times V_{i}}{C_{E T_{0}} \times V_{0}}\right) \times 100 \\
& F_{T}=\frac{\left|T_{o}-T_{i}\right|}{T_{o}} \times 100 \\
& F_{C}=\frac{\left(\frac{C_{E T_{0}} \times V_{0}-C_{E T_{i}} \times V_{i}}{V_{0}-V_{i}}\right)}{C_{E T_{0}}}
\end{aligned}
$$

\subsection{Método Analítico: Determinação do Teor Alcoólico}

O teor alcoólico das amostras foi determinado através da medida de densidade e expresso em ${ }^{\circ} \mathrm{GL}$ a $20{ }^{\circ} \mathrm{C}$, após destilação prévia de $25 \mathrm{~mL}$ da amostra em um microdestilador de álcool (modelo TE012, Tecnal). O teor alcoólico foi medido em densímetro digital (modelo DMA 48, Anton Paar).

\section{RESULTADOS E DISCUSSÃO}

Nos ensaios realizados, a temperatura do vinho (T) variou livremente ao longo do stripping, porém, a vazão específica de dióxido de carbono $\left(\Phi_{\mathrm{CO} 2}\right)$ permaneceu constante. Na Tabela 3 estão as matrizes do planejamento experimental com os valores dos parâmetros de desempenho obtidos após $8 \mathrm{~h}$ de stripping. 
Tabela 3 - Valores dos parâmetros de desempenho do processo de stripping.

\begin{tabular}{cccccc|cccccc}
\hline Ensaio & $\mathbf{X}_{\mathbf{1}}$ & $\mathbf{X}_{\mathbf{2}}$ & $\mathbf{F}_{\mathbf{A}}(\boldsymbol{\%})$ & $\mathbf{F}_{\mathbf{T}}(\boldsymbol{\%})$ & $\mathbf{F}_{\mathbf{C}}(-)$ & $\mathbf{E n s a i o}$ & $\mathbf{X}_{\mathbf{1}}$ & $\mathbf{X}_{\mathbf{3}}$ & $\mathbf{F}_{\mathbf{A}}(\boldsymbol{\%})$ & $\mathbf{F}_{\mathbf{T}}(\boldsymbol{\%})$ & $\mathbf{F}_{\mathbf{C}}(-)$ \\
\hline $\mathbf{1}$ & -1 & -1 & 3,37 & 10,30 & 12,77 & $\mathbf{8}$ & -1 & -1 & 11,15 & 14,24 & 8,37 \\
$\mathbf{2}$ & -1 & +1 & 4,11 & 12,42 & 9,77 & $\mathbf{9}$ & -1 & +1 & 18,18 & 14,85 & 9,84 \\
$\mathbf{3}$ & +1 & -1 & 8,16 & 30,15 & 5,20 & $\mathbf{1 0}$ & +1 & -1 & 24,91 & 43,54 & 5,41 \\
$\mathbf{4}$ & +1 & +1 & 16,30 & 35,85 & 5,76 & $\mathbf{1 1}$ & +1 & +1 & 39,14 & 46,77 & 6,33 \\
$\mathbf{5}$ & 0 & 0 & 5,75 & 22,86 & 5,46 & $\mathbf{1 2}$ & 0 & 0 & 23,53 & 30,41 & 6,42 \\
$\mathbf{6}$ & 0 & 0 & 6,12 & 24,49 & 5,83 & $\mathbf{1 3}$ & 0 & 0 & 22,96 & 38,37 & 6,41 \\
$\mathbf{7}$ & 0 & 0 & 5,98 & 23,47 & 5,14 & $\mathbf{1 4}$ & 0 & 0 & 23,26 & 39,39 & 6,66 \\
\hline
\end{tabular}

Examinando-se os dados de $\mathrm{F}_{\mathrm{A}}$ da Tabela 3, verifica-se que o maior valor para este parâmetro de desempenho foi de 39,14\% (Ensaio 11). A partir dos dados da Tabela 3 foram obtidos os diagramas de Pareto para o $\mathrm{F}_{\mathrm{A}}$ empregando faixas menores e maiores de $\Phi_{\mathrm{CO} 2}$, conforme ilustra a Figura 2. Adotou-se um intervalo de confiança de $90 \%$.

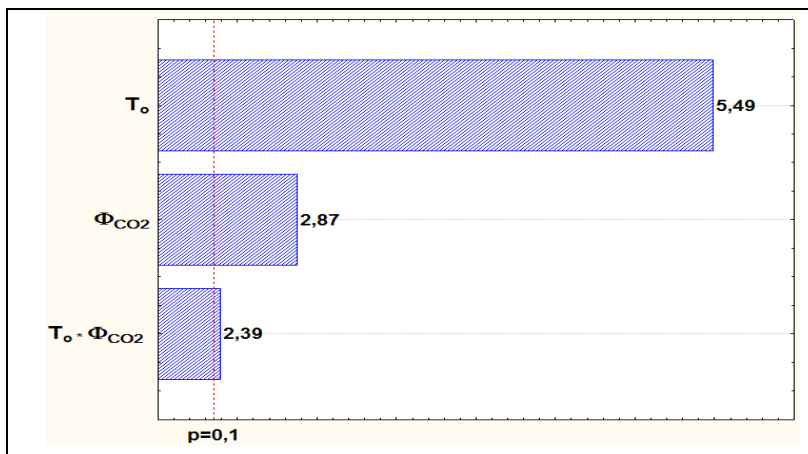

(a)

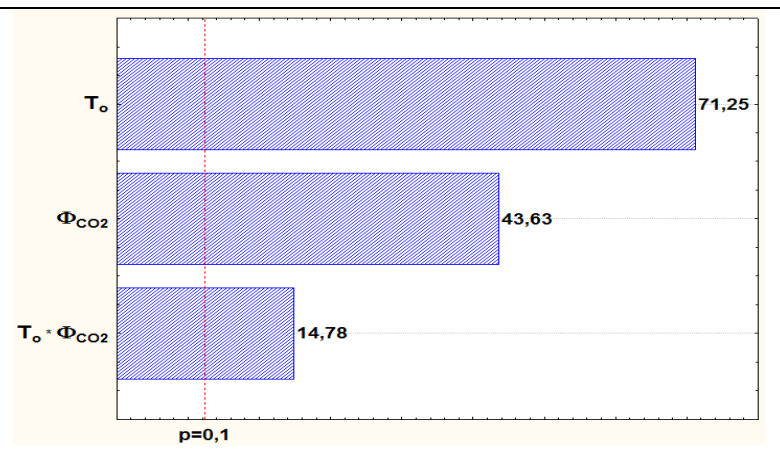

(b)

Figura 2 - Diagramas de Pareto das análises estatísticas para o $\mathrm{F}_{\mathrm{A}}$ empregando (a) menores $\Phi_{\mathrm{CO} 2}$ $\left(0,1\right.$ a 0,3 vvm) e (b) maiores $\Phi_{\mathrm{CO} 2}(0,5$ a 1,0 vvm).

Observa-se na Figura 2 que a $\mathrm{T}_{\mathrm{o}}$ foi a variável que mais influenciou o $\mathrm{F}_{\mathrm{A}}$, tendo um efeito positivo. Este resultado era esperado, pois com o aumento da $T_{0}$, a pressão de saturação do etanol se eleva e ocorre um aumento da fração de etanol na fase gasosa, gerando, portanto, uma maior vaporização. $\mathrm{O}$ aumento da $\mathrm{T}_{\mathrm{o}}$ provoca também uma redução na tensão superficial, pois com a maior energia cinética do meio, as forças intermoleculares ficam menos efetivas, facilitando a saída de moléculas de etanol do líquido para a fase gasosa, favorecendo a vaporização. Além disso, com a diminuição da tensão superficial, tem-se o aumento dos efeitos molhante, espalhante e penetrante do etanol, os quais contribuem para uma maior velocidade de absorção do etanol nas bolhas até que o equilíbrio seja atingido e, também, provavelmente auxiliam no arraste mecânico (sublação), pois se supõe que seja formada uma película líquida na superfície da bolha composta basicamente por etanol, por este possuir efeitos molhante e espalhante maiores do que a água. Observa-se, também, que a $\Phi_{\mathrm{CO} 2}$ apresentou um efeito positivo sobre o $\mathrm{F}_{\mathrm{A}}$, sendo, porém, menos significativa do que a $\mathrm{T}_{\mathrm{o}}$. Este resultado mostra-se coerente, pois ao se elevar a $\Phi_{\mathrm{CO} 2}$, ocorre um aumento na agitação do meio, diminuindo a espessura da película estagnada da fase líquida devido ao aumento do número de Reynolds e, com isso, tem-se um aumento da transferência de massa na película estagnada até que o equilíbrio entre as fases seja atingido. Além disso, há a fragmentação das bolhas de gás em um número maior de bolhas com menor diâmetro, aumentando, portanto, a área interfacial de transferência de 
massa, promovendo um maior arraste de massa de etanol. Além das variáveis isoladas, a interação entre as variáveis $T_{\mathrm{o}}$ e $\Phi_{\mathrm{CO} 2}$ também influenciou sobre o $\mathrm{F}_{\mathrm{A}}$.

Analisando-se os valores do $\mathrm{F}_{\mathrm{T}}$ na Tabela 3, verifica-se que houve a redução da temperatura do meio reacional em todos os experimentos, chegando a uma redução máxima de 46,8\% (Ensaio 11). Nos testes realizados a $33{ }^{\circ} \mathrm{C}$, Ensaios 1, 2, 8 e 9, o valor do $F_{T}$ variou de 10,3 a 14,9\%, a depender da $\Phi_{\mathrm{CO} 2}$. Sabendo-se que o processo fermentativo é exotérmico e que a temperatura adequada para a fermentação encontra-se entre 33 e $35{ }^{\circ} \mathrm{C}$, o stripping pode contribuir para o controle da temperatura da fermentação, o que seria vantajoso para as usinas, pois este controle é fundamental e bastante difícil. A partir dos dados da Tabela 3, foram obtidos os Diagramas de Pareto para o $\mathrm{F}_{\mathrm{T}}$ empregando-se faixas menores e maiores de $\Phi_{\mathrm{CO} 2}$, conforme ilustra a Figura 2. Adotou-se um intervalo de confiança de $90 \%$.

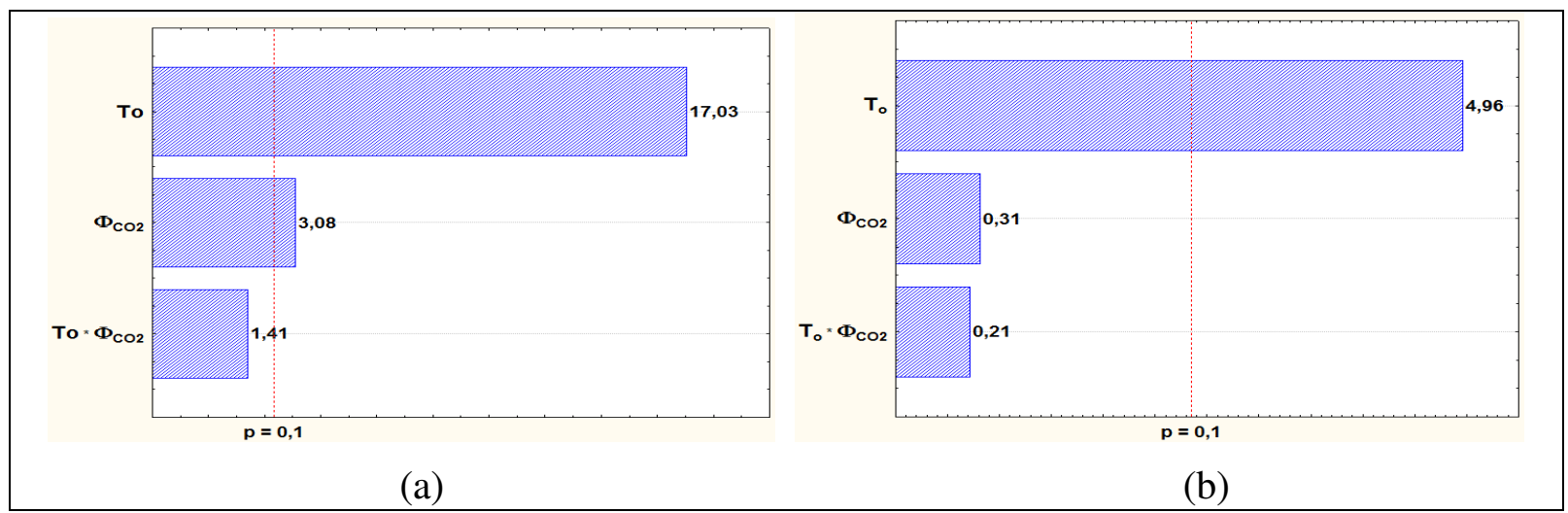

Figura 3 - Diagramas de Pareto para o $\mathrm{F}_{\mathrm{T}}$ empregando (a) menores $\Phi_{\mathrm{CO} 2}(0,1$ a $0,3 \mathrm{vvm})$ e (b) maiores $\Phi_{\mathrm{CO} 2}(0,5$ a $1,0 \mathrm{vvm})$.

Na Figura 3 verifica-se que a $T_{0}$ foi a variável independente de maior influência no $F_{T}$, tendo um efeito positivo. Esse comportamento ocorre, pois, quando se inicia o processo de stripping a uma $\mathrm{T}_{\mathrm{o}}$ maior, aumenta-se a pressão de saturação do meio e, consequentemente, ocorre uma maior vaporização, retirando-se, com isso, uma maior quantidade de energia do sistema e, portanto, diminuindo a sua temperatura. Além da $\mathrm{T}_{\mathrm{o}}$, nota-se que o $\mathrm{F}_{\mathrm{T}}$ foi influenciado positivamente pela $\Phi_{\mathrm{CO} 2}$, porém, apenas quando se trabalhou em faixas menores de $\Phi_{\mathrm{CO} 2}$ (Figura 3(a)). O aumento da $\Phi_{\mathrm{CO} 2}$ implica em um maior arraste de etanol, conforme observa-se na Figura 2. No entanto, há dois tipos de arraste, o arraste termodinâmico (vaporização) e o arraste mecânico (sublação), sendo que, a vaporização proporciona a diminuição da temperatura do meio, enquanto que na sublação, as moléculas do vinho são retiradas na forma líquida e, consequentemente, não ocorre a remoção de calor do vinho. Conforme foi apresentado na Figura 3(b), a variável $\Phi_{\mathrm{CO} 2}$ não foi significativa para o $\mathrm{F}_{\mathrm{T}}$, podendo-se supor que, neste caso, o equilíbrio não teria sido atingido, pois o tempo de residência não foi suficiente para que as bolhas de $\mathrm{CO}_{2}$ ficassem saturadas e, com isso, carregassem consigo menores quantidades de vapores do que no equilíbrio, o que acarretou em uma menor retirada de energia do sistema, sendo que o maior arraste de etanol observado, através do $\mathrm{F}_{\mathrm{A}}$, com o aumento da $\Phi_{\mathrm{CO} 2}$ (Figura 3(b)) pode ser atribuído a sublação, devido à maior área de contato, fazendo com que o etanol molhe e se espalhe na superfície das bolhas e seja arrastado na forma líquida, sem retirar calor do meio. Para explicar a influência da variável $\Phi_{\mathrm{CO} 2}$, observada na Figura 3(a), sobre o $\mathrm{F}_{\mathrm{T}}$, pode-se admitir que, quando utilizadas faixas menores de $\Phi_{\mathrm{CO} 2}$, o tempo de residência é suficiente para que as bolhas de $\mathrm{CO}_{2}$ fiquem saturadas, ou seja, em equilíbrio, 
gerando o arraste através da vaporização, retirando energia do meio. Supõe-se que haja também o arraste mecânico, porém, com pequena intensidade, pois tem-se um menor número de bolhas com maior diâmetro, o que diminui a área de contato entre o vinho e as bolhas. Este resultado é interessante, pois se o stripping fosse utilizado apenas para auxiliar no controle de temperatura das dornas, o ideal seria utilizar a menor $\Phi_{\mathrm{CO} 2}$ (de modo a atingir o equilíbrio), para que o etanol seja arrastado principalmente pela vaporização, pois a sublação não interfere na temperatura do meio.

A Figura 4 mostra os Diagramas de Pareto para o $F_{C}$ utilizando faixas menores e maiores $\Phi_{\mathrm{CO} 2}$ (intervalo de confiança de $90 \%$ ). Na Figura verifica-se que o valor da $\mathrm{T}_{\mathrm{o}}$ influenciou no $\mathrm{F}_{\mathrm{C}}$, indicando que, com o aumento da $\mathrm{T}_{\mathrm{o}}$, ocorre o arraste de uma maior quantidade de água juntamente com o etanol, gerando uma corrente mais diluída.

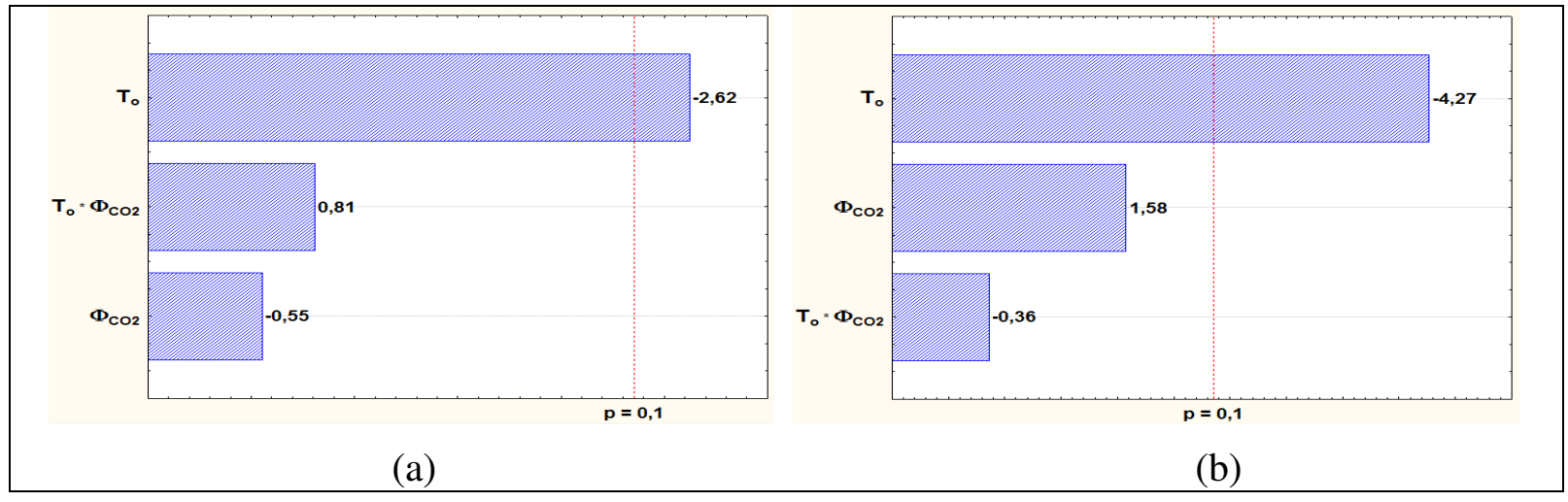

Figura 4 - Diagramas de Pareto das análises estatísticas para o $\mathrm{F}_{\mathrm{C}}$ empregando (a) menores $\Phi_{\mathrm{CO} 2}$ $(0,1$ a $0,3 \mathrm{vvm})$ e (b) maiores $\Phi_{\mathrm{CO} 2}(0,5$ a $1,0 \mathrm{vvm})$

\section{CONCLUSÕES}

$O \mathrm{~F}_{\mathrm{A}}$ mostrou-se fortemente influenciado pela $\mathrm{T}_{\mathrm{o}}$, pois eleva-se a pressão de saturação da fase líquida e provoca-se a redução da tensão superficial. A $\Phi_{\mathrm{CO} 2}$ apresentou um efeito positivo sobre o $\mathrm{F}_{\mathrm{A}}$, devido a maior agitação do meio, o que provoca um aumento da transferência de massa entre as fases e da área de contato entre as bolhas e o vinho, contribuindo, também, para o arraste de etanol. $\mathrm{O} \mathrm{F}_{\mathrm{T}}$ foi fortemente influenciado positivamente pela $\mathrm{T}_{\mathrm{o}}$, pois ocorre uma maior vaporização em temperaturas maiores, retirando uma maior quantidade de energia do sistema. A $\Phi_{\mathrm{CO} 2}$ teve influência positiva sobre o $\mathrm{F}_{\mathrm{T}}$, porém, apenas para as faixas menores de $\Phi_{\mathrm{CO} 2}$. Supõe-se que, para faixas maiores de $\Phi_{\mathrm{CO} 2}$ houve menor tempo de residência das bolhas no vinho e, com isso, o equilíbrio não foi atingido, acarretando em uma menor retirada de energia do sistema, sendo que o maior arraste de etanol com o aumento da vazão, para as faixas maiores de $\Phi_{\mathrm{CO} 2}$, mostrado através do $\mathrm{F}_{\mathrm{A}}$, pode ser atribuído a sublação que não remove calor do meio. $\mathrm{O} \mathrm{F}_{\mathrm{C}}$ foi influenciado negativamente pela variável $\mathrm{T}_{\mathrm{o}}$, pois ocorre o arraste de uma maior quantidade de água juntamente com o etanol.

\section{AGRADECIMENTOS}

À Virgolino de Oliveira S/A e à FAPESP (Projeto PITE - Processo 2012/50046-4). 


\section{NOMENCLATURA}

$\mathrm{C}_{\mathrm{ETo}}$ : concentração de etanol inicial na fase líquida $\left(\mathrm{g} \cdot \mathrm{L}^{-1}\right)$;

$\mathrm{C}_{\mathrm{ETi}}$ : concentração de etanol no instante $\mathrm{t}=\mathrm{i}$ na fase líquida $\left(\mathrm{g} \cdot \mathrm{L}^{-1}\right)$;

$\mathrm{m}_{\mathrm{ETo}}$ : massa de etanol inicial na fase líquida $(\mathrm{g})$;

$\mathrm{m}_{\mathrm{ETi}}$ : massa de etanol no instante $\mathrm{t}=\mathrm{i}$ na fase líquida $(\mathrm{g})$;

$\mathrm{T}_{\mathrm{o}} \quad$ : temperatura inicial do vinho $\left({ }^{\circ} \mathrm{C}\right)$;

$\mathrm{T}_{\mathrm{i}}$ : temperatura do vinho no instante $\mathrm{i}$ ao longo do experimento $\left({ }^{\circ} \mathrm{C}\right)$;

$\mathrm{V}_{\mathrm{o}}$ : volume inicial da fase líquida (L);

$\mathrm{V}_{\mathrm{i}}$ : volume no instante $\mathrm{t}=\mathrm{i}$ na fase líquida $(\mathrm{L})$.

\section{REFERÊNCIAS}

AMORIM, H. V.; LOPES, M. L.; OLIVEIRA, J. V. C.; BUCKERIDGE, M. S.; GOLDMAN, G. H. Scientific challenges of bioethanol production in Brazil. Applied Microbiology and Biotechnology, v. 91, p. 1267-1275, 2011.

ANP, Especificações Hidratado Carburante. http://www.scalcool.com.br/arquivos/docs/2012/05/6606eb3b1a047cd35059381295023796. pdf (Acessado 22/04/2014).

ESPERANÇA, M. N.; CRUZ, A. J. G.; MOURA, L. F.; SILVA, C. R.; BADINO, A. C.. Avaliação das influências de variáveis de operação no arraste de etanol por $\mathrm{CO}_{2}$. Anais do Congresso Brasileiro de Engenharia Química, 2012.

MORANDIN, M.; TOFFOLO, A.; LAZZARETTO, A.; MARECHAL, F., ENSINAS, A. V.; NEBRA, S. A. Synthesis and parameter optimization of a combined sugar and ethanol production process integrated with a CHP system. Energy, v. 36, p. 3675-3690, 2011.

RIVERA, E. C.; ATALA, D. I. P.; MAUGERI, F.; COSTA, A. C.; MACIEL, R. Development of real-time state estimators for reaction-separation processes: A continuous flash fermentation as a study case. Chemical Engineering and Processing, v. 49, p. 402-409, 2010.

RODRIGUES, M. I.; IEMMA, A. F. Planejamento de experimentos e otimização de processos: uma sequencia de planejamentos. Editora Casa do Pão, 2009.

SCHUGERL, K. Integrated processing of biotechnology products. Biotechnology Advances, v. 18, p. 581-599, 2000.

TAYLOR, F.; KURANTZ, M. J.; GOLDBERG, N.; CRAIG JR, J. C. Effects of ethanol concentration and stripping temperature on continuous fermentation rate. Applied Microbiology and Biotechnology, v. 48, p. 311-316, 1997.

UNICA: http://www.unica.com.br/noticia/1863142892039218077/campanha-publicitaria-porcentoE2-por-cento80-por-cento9Cetanol-por-cento2C-o-combustivel/

(Acessado 02/04/2014). 